\title{
Progetto SLAM: un approccio multidisciplinare al follow-up di secondo livello nella gestione delle comorbidità nel paziente HIV positivo. Valutazione del danno cardiovascolare SLAM Project: a multidisciplinary approach to the second level follow-up in the management of comorbidities in HIV-positive patients. Evaluation of cardiovascular diseaese
}

\author{
Tiziana Quirino ${ }^{1}$, Barbara Menzaghi \\ ${ }^{1}$ Coordinamento Gruppo C.I.S.A.I. \\ ${ }^{2}$ ASST Valle Olona U.O. Malattie Infettive, Busto Arsizio (VA)
}

\section{Riassunto}

Le malattie cardiovascolari rappresentano una delle principali cause di morte per eventi non-AIDS nella popolazione con infezione da HIV verosimilmente in conseguenza ad un incremento dei fattori di rischio cardiovascolare rispetto alla popolazione generale e ad un danno legato al processo infiammatorio secondario all'infezione virale.

È evidente quindi che diventa estremamente importante un monitoraggio accurato dei fattori che incrementano il rischio cardiovascolare e degli strumenti di diagnosi precoce. Presentiamo i risultati relativi alla valutazione del danno cardiovascolare che abbiamo ottenuto nell'ambito di uno studio più completo che intende proporre un assessment diagnostico multidimensionale di secondo livello sulle principali comorbidità in corso di infezione da HIV. Per la realizzazione del nostro studio abbiamo utilizzato la metodologia Delphi.

Abbiamo identificato e sottomesso il questionario ad un panel ristretto di 48 specialisti esperti di 10 Centri Italiani di diagnosi e cura dell'infezione da HIV chiedendo di valutare l'utilità del test, la sua disponibilità presso il Centro di appartenenza e l'eventuale necessità di una prescrizione specialistica.

I risultati ottenuti sono stati confrontati con le indicazioni delle Linee Guida Italiane 2017.

\section{Abstract}

Cardiovascular disease is one of the main causes of death for non-AIDS-related events among the HIV-infected population. Recent meta-analysis studies including both observational studies and randomized trials, report that the onset of cardiovascular diseases is higher in HIV-positive subjects compared to the general population, both among ARV-treated and untreated patients.

Reasons for this are well known: in HIV-infected subjects there are increased cardiovascular risk factors, combined with cardiovascular damage caused by HIV directly. Antiretroviral treatment itself can play a part in causing cardiovascular disease. It is therefore extremely important, in view of documented efficacy of the antiretroviral treatment, to accurately monitor those subjective factors that can increase cardiovascular risk, and to use tools for early diagnosis. We intend to present data from a survey conducted among infectious diseases specialists who were asked to identify which tests they deemed essential to monitor cardiovascular damage. This information was obtained as part of a more comprehensive study that aimed to offer a second-level multidimensional diagnostic assessment regarding the main comorbidities in HIV-infection. Examining the preferences expressed by physicians when completing the survey, a substantial adherence to the Italian guidelines is shown. Most of the assessments for which an agreement was not reached are in fact indicated in the guidelines with a Ilb level of evidence.

\section{Introduzione}

Le malattie cardiovascolari rappresentano una delle principali cause di morte per eventi non-
AIDS nella popolazione con infezione da HIV. Recenti studi di meta-analisi che hanno incluso studi osservazionali e trial randomizzati, hanno riportato che
Autore

per la corrispondenza:

Tiziana Quirino

Coordinamento Gruppo

C.I.S.A.I.

tiziana.quirino@gmail.com

Keywords:

HIV; Cardiovascular diseases; Delphi method.

Potenziali conflitti di interesse:

nessuno.

JHA 2018; S1: 15-19

DOI: 10.19198/JHA31465 
la comparsa di malattia cardiovascolare è più elevata rispetto alla popolazione generale sia nei pazienti trattati con terapia antiretrovirale che nei non trattati. Le cause di questo sono note. Infatti nei soggetti con infezione da HIV è presente un incremento dei noti fattori di rischio cardiovascolare a cui si aggiunge il danno legato ad HIV.

Il trattamento antiretrovirale può, a sua volta, giocare un ruolo nel determinare la patologia cardiovascolare. $\grave{E}$ evidente quindi che diventa estremamente importante, a fronte di una documentata efficacia del trattamento antiretrovirale, un monitoraggio accurato dei fattori soggettivi che incrementano il rischio cardiovascolare e l'utilizzo di strumenti di diagnosi precoce.

Intendiamo presentare i risultati relativi ad un'indagine condotta presso specialisti infettivologi a cui è stato chiesto di identificare quali esami fossero indispensabili nel monitoraggio del danno cardiovascolare. Tali informazioni sono state ottenute nell'ambito di uno studio più completo che intende proporre un assessment diagnostico multidimensionale di secondo livello relativamente alle principali comorbidità in corso di infezione da HIV.

Considerando le priorità indicate dai clinici nella compilazione del questionario si riscontra una sostanziale adesione alle indicazioni delle Linee guida italiane. La maggior parte degli esami che non hanno raggiunto l'agreement sono infatti indicati nelle Linee guida con evidenza BII.

\section{Materiali e metodi}

Lo studio ha valutato e definito una proposta di esami da effettuare come screening di secondo livello nell'ambito di una strategia di monitoraggio delle comorbidità già indagata in screening di primo livello da altre ricerche (11). Questa seconda valutazione consente di indicare il necessario approfondimento diagnostico nel caso in cui lo specialista infettivologo rilevi la presenza di fattori di rischio e/o di comorbidità già attive nel paziente HIV. Per la realizzazione del nostro studio abbiamo utilizzato la metodologia Delphi.

Lo studio è stato condotto dal Gruppo CISAI (Coordinamento Italiano per lo Studio dell'Allergia in Infezione da HIV) e a tale fine è stato costituito un Comitato scientifico composto da quattro esperti di livello nazionale nella gestione di studi clinici sulla patologia HIV correlata.
Per la definizione del questionario da sottoporre agli specialisti abbiamo analizzato gli item di secondo livello che erano già stati individuati dal Panel che si è occupato dello studio di primo livello ma che non erano ancora stati sottoposti a clinici (11). Questi item sono stati rivisti e in parte modificati dal Comitato scientifico. Inoltre è stata valutata la reale disponibilità degli esami proposti presso i Centri che hanno partecipato alla rilevazione e valutato l'orientamento degli infettivologi verso il coinvolgimento e la condivisione del paziente con gli altri specialisti indicando le condizioni in cui debba essere attivata una gestione multidisciplinare della comorbidità.

Abbiamo identificato e sottomesso il questionario ad un panel ristretto di 48 esperti che prestano la loro attività in 10 Centri Italiani di diagnosi e cura dell'infezione da HIV.

Gli specialisti sono stati selezionati secondo criteri di competenza ed esperienza nel settore. II panel è quindi rappresentativo della realtà nazionale ed esprime una importante esperienza pluriennale nel settore.

Tra il mese di marzo ed il mese di luglio del 2018 il questionario è stato somministrato ai clinici in due fasi successive; al termine della prima fase sono stati definiti gli item che avevano raggiunto l'agreement, quelli per cui era emerso disagreement e quelli su cui i pareri erano dispersi. Questi ultimi sono stati sottoposti ad una nuova valutazione da parte dei clinici con lo stesso metodo seguito in precedenza. Ognuno dei partecipanti ha avuto acceso ad una piattaforma web dedicata al progetto, ha ricevuto tutte le informazioni relative al progetto stesso, la selezione della bibliografia, la sinossi dello studio e la descrizione del Metodo Delphi da applicare. II questionario era costituito da 102 voci suddivise in 7 aree di indagine: Diagnostica di area Infettivologica, Diagnostica Osteoarticolare, Diagnostica Metabolica e Cardiovascolare, Diagnostica Nefrologica, Diagnostica Epatologica, Diagnostica del Sistema Nervoso Centrale e Diagnostica Psicologica e Qualità della Vita.

Le risposte erano distribuite su una scala Likert da 1 a 9, dove 1 = sicuramente non utile e 9 = sicuramente utile. Valori intermedi corrispondono a differenti modulazioni rispettivamente del giudizio di non utilità (2-3), di utilità dubbia (4-6), e di utilità (7-9).

Abbiamo concluso per:

- agreement se I'80\% dei pareri era all'interno di uno dei tre punteggi $1-3,4-6,7-9$ 
- disagreement se il $90 \%$ dei pareri era all'interno dei punteggi 1-6 or 4-9

\section{Risultati}

Abbiamo analizzato i risultati dei 48 questionari compilati per la parte relativa alla diagnostica cardiovascolare di secondo livello.

Tra il primo ed il secondo round non ci sono state variazioni nelle risposte agli item riproposti; si è quindi deciso di non procedere ad un terzo round di valutazioni.

I risultati complessivi del percorso di consenso sono riportati in tabella 1.
Raggiungono l'agreement solo: Hb glicata, ASCVD risk score, ECG e valutazione dell'intima media thickness (IMT). Disagreement è espresso per la sola insulinemia; per i restanti item si registrano pareri dispersi.

Per 3 dei 4 item con agreement era riferita una disponibilità del 100\%, mentre IMT era disponibile nel 75\% dei casi. Insulinemia, era disponibile nel 93,8\%.

Gli item con pareri dispersi erano in tre casi disponibili oltre il $90 \%$ e nei restanti inferiori al $50 \%$. Per quanto riguarda l'identificazione della figura professionale che dovrebbe fornire indicazione all'esame, l'infettivologo è stato indicato da oltre

\begin{tabular}{|c|c|c|c|c|c|c|c|c|c|c|c|c|}
\hline \multicolumn{2}{|l|}{$\begin{array}{l}\text { DIAGNOSTICA } \\
\text { CARDIOVASCOLARE }\end{array}$} & \multicolumn{6}{|c|}{ ANALISI } & \multirow{2}{*}{$\begin{array}{c}\text { UTILITÀ } \\
\begin{array}{c}\text { Livello } \\
\text { di consenso }\end{array}\end{array}$} & \multicolumn{2}{|c|}{ DISPONIBILITÀ } & \multicolumn{2}{|c|}{ INDICAZIONE } \\
\hline $\begin{array}{l}\text { DIAGNOSTICA } \\
\text { CARDIOVASCOLARE }\end{array}$ & $\begin{array}{l}\text { ITEM da } \\
\text { valutare }\end{array}$ & $\mathbf{n}$ & $\begin{array}{c}\% \\
1-3\end{array}$ & $\begin{array}{c}\% \\
4-6\end{array}$ & $\begin{array}{c}\% \\
7-9\end{array}$ & $\begin{array}{c}\% \\
4-9\end{array}$ & $\begin{array}{c}\% \\
1-6\end{array}$ & & Disponibile & $\begin{array}{c}\text { Non } \\
\text { Disponibile }\end{array}$ & Infettivologo & Specialista \\
\hline $\begin{array}{l}\text { FATTORI } \\
\text { DI RISCHIO PER CVD }\end{array}$ & $\mathrm{Hb}$ glicata & 48 & $2,1 \%$ & $14,6 \%$ & $83,3 \%$ & $97,9 \%$ & $16,7 \%$ & AGREEMENT & $100,0 \%$ & $0,0 \%$ & $75,0 \%$ & $25,0 \%$ \\
\hline $\begin{array}{l}\text { STIMA } \\
\text { DEL RISCHIO CVD }\end{array}$ & $\begin{array}{l}\text { ASCVD risk } \\
\text { score }\end{array}$ & 48 & $2,1 \%$ & $10,4 \%$ & $87,5 \%$ & $97,9 \%$ & $12,5 \%$ & AGREEMENT & $100,0 \%$ & $0,0 \%$ & $70,8 \%$ & $29,2 \%$ \\
\hline $\begin{array}{l}\text { VALUTAZIONE } \\
\text { DANNO VASCOLARE }\end{array}$ & ECG & 48 & $0,0 \%$ & $14,6 \%$ & $85,4 \%$ & $100,0 \%$ & $14,6 \%$ & AGREEMENT & $100,0 \%$ & $0,0 \%$ & $77,1 \%$ & $22,9 \%$ \\
\hline $\begin{array}{l}\text { VALUTAZIONE } \\
\text { DANNO VASCOLARE }\end{array}$ & IMT & 48 & $0,0 \%$ & $18,8 \%$ & $81,3 \%$ & $100,0 \%$ & $18,8 \%$ & AGREEMENT & $75,0 \%$ & $25,0 \%$ & $52,1 \%$ & $47,9 \%$ \\
\hline $\begin{array}{l}\text { STIMA } \\
\text { DEL RISCHIO CVD }\end{array}$ & Insulinemia & 48 & $8,3 \%$ & $45,8 \%$ & $45,8 \%$ & $91,7 \%$ & $54,2 \%$ & DISAGREEMENT & $93,8 \%$ & $6,3 \%$ & $41,7 \%$ & $58,3 \%$ \\
\hline $\begin{array}{l}\text { STIMA } \\
\text { DEL RISCHIO CVD }\end{array}$ & IL-6 & 48 & $39,6 \%$ & $37,5 \%$ & $22,9 \%$ & $60,4 \%$ & $77,1 \%$ & PARERI DISPERSI & $29,2 \%$ & $70,8 \%$ & $45,8 \%$ & $54,2 \%$ \\
\hline $\begin{array}{l}\text { STIMA } \\
\text { DEL RISCHIO CVD }\end{array}$ & hsPCR & 48 & $18,8 \%$ & $50,0 \%$ & $31,3 \%$ & $81,3 \%$ & $68,8 \%$ & PARERI DISPERSI & $39,6 \%$ & $60,4 \%$ & $43,8 \%$ & $56,3 \%$ \\
\hline $\begin{array}{l}\text { STIMA } \\
\text { DEL RISCHIO CVD }\end{array}$ & Fibrinogeno & 48 & $18,8 \%$ & $43,8 \%$ & $37,5 \%$ & $81,3 \%$ & $62,5 \%$ & PARERI DISPERSI & $97,9 \%$ & $2,1 \%$ & $62,5 \%$ & $37,5 \%$ \\
\hline $\begin{array}{l}\text { STIMA } \\
\text { DEL RISCHIO CVD }\end{array}$ & D-dimero & 48 & $25,0 \%$ & $31,3 \%$ & $43,8 \%$ & $75,0 \%$ & $56,3 \%$ & PARERI DISPERSI & $100,0 \%$ & $0,0 \%$ & $52,1 \%$ & $47,9 \%$ \\
\hline $\begin{array}{l}\text { STIMA } \\
\text { DEL RISCHIO CVD }\end{array}$ & Omocisteina & 48 & $16,7 \%$ & $31,3 \%$ & $52,1 \%$ & $83,3 \%$ & $47,9 \%$ & PARERI DISPERSI & $97,9 \%$ & $2,1 \%$ & $54,2 \%$ & $45,8 \%$ \\
\hline $\begin{array}{l}\text { VALUTAZIONE } \\
\text { DANNO VASCOLARE }\end{array}$ & $\begin{array}{c}\text { Test } \\
\text { endoteliale } \\
\text { flusso } \\
\text { mediato }\end{array}$ & 48 & $18,8 \%$ & $43,8 \%$ & $37,5 \%$ & $81,3 \%$ & $62,5 \%$ & PARERI DISPERSI & $18,8 \%$ & $81,3 \%$ & $18,8 \%$ & $81,3 \%$ \\
\hline $\begin{array}{l}\text { VALUTAZIONE } \\
\text { DANNO VASCOLARE }\end{array}$ & $\begin{array}{l}\text { Indice } \\
\text { pressorio } \\
\text { caviglia } \\
\text { braccio }\end{array}$ & 48 & $10,4 \%$ & $47,9 \%$ & $41,7 \%$ & $89,6 \%$ & $58,3 \%$ & PARERI DISPERSI & $41,7 \%$ & $58,3 \%$ & $16,7 \%$ & $83,3 \%$ \\
\hline $\begin{array}{l}\text { VALUTAZIONE } \\
\text { DANNO VASCOLARE }\end{array}$ & CAC score & 48 & $18,8 \%$ & $41,7 \%$ & $39,6 \%$ & $81,3 \%$ & $60,4 \%$ & PARERI DISPERSI & $43,8 \%$ & $56,3 \%$ & $14,6 \%$ & $85,4 \%$ \\
\hline
\end{tabular}

Tabella 1. Risultati relativi alla diagnostica cardiovascolare di secondo livello 
il 50\% dei partecipanti per la metà degli esami; in particolare $\mathrm{Hb}$ glicata, ASCVD risk score ed ECG erano indicati a prescrizione infettivologica in oltre il $70 \%$ dei casi.

\section{Discussione}

Dalla valutazione dei risultati sono possibili alcune considerazioni. Esaminando le risposte per tipologia di diagnostica vediamo come fra gli esami proposti per la valutazione del rischio cardiovascolare solo ASCVD risk score abbia ricevuto agreement. Gli altri item non hanno raggiunto una valutazione positiva nonostante la maggior parte di questi siano ampiamente disponibili nei centri; in particolare insulinemia, fibrinogeno, D-dimero, omocisteina con valori > al $90 \%$.

Inoltre circa la metà dei partecipanti ritiene che questi esami debbano essere richiesti dal cardiologo. Per quanto riguarda la valutazione del danno vascolare, solo IMT ha raggiunto l'agreement, con una disponibilità dell'esame pari al 75\% e una indicazione alla prescrizione dell'esame a carico dell'infettivologo solo nel 52\% dei casi.

Il test endoteliale flusso mediato, l'indice pressorio caviglia braccio e il CAC score non hanno raggiunto agreement; è riferita una disponibilità ridotta e in oltre l'80\% dei casi la prescrizione del test è indicata come a carico del cardiologo.

Al fine di conoscere quanto queste valutazioni siano aderenti alle Linee Guida Nazionali, le abbiamo confrontate con quanto indicato nella versione del 2017 (12).

In relazione alla stima del rischio, le Linee guida 2017 indicano l'esecuzione di ECG e la valutazione di ASCVD risk score con raccomandazione All. L'esecuzione dei markers di infiammazione, IL6 - hsPCR - fibrinogeno - D-dimero e omocisteina presenta una raccomandazione BII; viene inoltre specificato che "lo studio di tali marcatori è al momento da riservarsi nell'ambito della ricerca" Nelle Linee guida si parla poi di valutazione del danno vascolare nell'ambito della "valutazione individuale di vulnerabilità" indicando sia IMT che CAC score con raccomandazione Bll quindi non consigliate ma da considerare. Si specifica che tali esami " hanno dimostrato di essere in grado di predire gli eventi cardiovascolari in maniera indipendente dai fattori di rischio classici, pertanto possono essere utilizzati per riclassificare il rischio cardiovascolare intermedio".
Come test di danno funzionale viene indicato indice pressorio caviglia-braccio sempre segnalato come Bll. Considerando le priorità indicate dai clinici nella compilazione del questionario si riscontra una sostanziale adesione alle Linee guida italiane. La maggior parte degli esami che sono stati valutati con "pareri dispersi" sono infatti indicati nelle Linee guida con evidenza B quindi come controlli da considerare ma non fortemente consigliati. È probabile che la "dispersione dei pareri" sia conseguenza delle caratteristiche Centri di Malattie Infettive di appartenenza, forse più che non della disponibilità dell'esame e del fatto che vi si svolga attività di assistenza oppure anche di ricerca clinica. In generale comunque le valutazioni di agreement e "non agreement" sembrano riflettere coerentemente quello che serve ad inquadrare in modo strettamente operativo il problema del paziente e a orientare le ulteriori indagini o le possibili soluzioni.

È verosimile che l'infettivologo, pur tenendo evidentemente in grande considerazione il problema delle comorbidità, senta la necessità, almeno nella pratica clinica, di un approccio pratico e multidisciplinare garantendo in questo modo una migliore appropriatezza prescrittiva.

Se queste considerazioni saranno ulteriormente confermate nell'ambito di necessari dibattiti e approfondimenti, potranno costituire un'ulteriore indicazione nella stesura delle Linee guida nazionali.

\section{Ringraziamenti:}

Barbara Belfiori, Chiara Bellacosa, Paolo Bonfanti, Giorgio Bozzi, Gabriella Chieffo, Elisa Colella, Paola Corsi, Giuseppe De Socio, Chiara Dentone, Mariana Farenga, Vito Fiore, Marco Franzetti, Francesco Maria Fusco, Chiara Gabrielli, Marta Guastavigna, Sergio Lo Caputo, Carla Lovigu,, Giordano Madeddu, Paolo Maggi, Marco Mannazzu, Letizia Marinaro, Canio Martinelli, Giovanni Mazzarello, Chiara Molteni, Laura Ambra Nicolini, Silvia Nozza, Giancarlo Orofino, Alessandro Pandolfo, Donatella Penoncelli, Piera Pierotti, Marco Pozzi, Daniele Rosignoli, Francesca Sabbatini, Elena Salomoni, Elisabetta Schiaroli, Nicola Squillace, Lucia Taramasso, Alessandro Tebini, Francesca Vichi, Raffaella Visonà, Paola Vitiello.

Finanziamenti: Gilead unrestricted grant. 


\section{BIBLIOGRAFIA}

1. Mocroft, A, Reiss P, Gasiorowski J et al., Serious fatal and nonfatal non-AIDS-defining illnesses in Europe. J Acquir Immune Defic Syndr 2010; 55:262-70.

2. Palella FJ, Baker RK, Moorman AC, et al. Mortality in the highly active antiretroviral therapy era: changing causes of death and disease in the HIV outpatient study. J Acquir Immune Defic Syndr 2006; 43: 27-34.

3. Lewden C, May T, Rosenthal E, et al. Changes in causes of death among adults infected by HIV between 2000 and 2005: The "Mortalité 2000 and 2005" surveys (ANRS EN19 and Mortavic). J Acquir Immune Defic Syndr 2008; 48: 590-8.

4. Islam FM, Wu J, Jansson J, et al. Relative risk of cardiovascular disease among people living with HIV: a systematic review and metaanalysis. HIV Med 2012;13:453-68.

5. Freiberg MS, Chang C-CH, Kuller LH, et al. HIV infection and the risk of acute myocardial infarction. JAMA Intern Med 2013;173:614-22.

6. Rickerts V, Brodt H, Staszewski S, et al. Incidence of myocardial infarctions in HIV-infected patients between 1983 and 1998:the Frankfurt HIV-cohort study. Eur J Med Res 2000; 5: 329-33.

7. Coll B, Parra S, Alonso-Villaverde C, et al. The role of immunity and inflammation in the progression of atherosclerosis in patients with HIV infection. Stroke 2007; 38: 2477-84.

8. Stein JH. Managing cardiovascular risk in patients with HIV infection. J Acquir Immune Defic Syndr 2005; 38: 115-23.

9. Strategies for Management of Antiretroviral Therapy (SMART) Study Group. Major clinical outcomes in antiretroviral the-rapy (ART)-naive participants and in those not receiving ART at baseline in the SMART study. J Infect Dis 2008; 197: 1133-44.

10. DAD Study Group. Use of nucleoside reverse transcriptase inhibitors and risk of myocardial infarction in HIV-infected patients enrolled in the D:A:D study: a multi-cohort collaboration. Lancet 2008; 371: 1417-26.

11. Borderi M, Angarano G, Antinori A et al. Managing the long surviving HIV patient: a proposal for a multidimensional first-level diagnostic assessment - New Microbiol. 2018; 41:112-117.

12. Linee Guida Italiane sull'utilizzo della Terapia Antiretrovirale e la gestione diagnostico-clinica delle persone con infezione da HIV-1 SIMIT 2017. Ultimo accesso 17 novembre 2018. 